\title{
Mössbauer Spectroscopy, Superparamagnetism and Ferrofluids
}

\author{
H.-D. Pfannes, J. H. Dias Filho, R. Magalhães-Paniago, \\ J. L. López, and R. Paniago \\ Laboratory for Hyperfine Spectroscopy and Surface Physics \\ Departamento de Física, Universidade Federal de Minas Gerais, Belo Horizonte, Brazil
}

Received on 21 December, 2000

\begin{abstract}
Some fundamentals of Mössbauer spectroscopy and of fluctuating magnetic hyperfine interactions are reviewed. An expression for calculation of spin-phonon interaction transition probabilities on the ground of a Debye model is given. Basic ideas of superparamagnetism and classical results for low-temperature relaxation rates are presented. A theory of superparamagnetism based on spinphonon interaction is introduced. It is shown how to calculate from this Mössbauer spectra taking all spin-levels into account. Experimental spectra of ferrofluids are compared with simulated spectra and found in good accordance.
\end{abstract}

\section{Introduction}

The Mössbauer effect is based on the "recoilless" absorption and emission of low energy $\left(E_{0}=10-100 \mathrm{keV}\right)$ $\gamma$-photons in solids $[1,2]$. A simple explanation for this effect relies on the Heisenberg uncertainty relation: the uncertainty in momentum $\Delta p \approx \hbar / \Delta x$ of bound atoms is of the order of $10^{-23} \mathrm{kgms}^{-1}(\Delta x=0.1 \AA)$ but the recoil moment of e.g. a $\gamma$-photon with energy $E_{0}=10 \mathrm{keV}$ is smaller $\left(\hbar \approx 5 \cdot 10^{-24} \mathrm{kgms}^{-1}\right)$, i.e. the recoil which eventually can excite vibrations (phonons) of the emitting atom sometimes may not be measurable. The recoil is then transmitted to the crystal as a whole and since the mass of the crystal is much greater than the atomic mass it does not alter the $\gamma$-energy ("recoilless" emission). The Mössbauer radiation emitted from a Mössbauer source has therefore the exact resonance energy necessary for absorption in an absorber containing the same material as the source. Moreover, when the lifetime $\tau$ of the excited Mössbauer energy level is longer (e.g. $\tau=10^{-7} \mathrm{~s}$ ) than the inverse vibration (phonon) frequency of the emitting or absorbing atom (e.g. $10^{-13} \mathrm{~s}$ ) the atom carries out many cycles during the lifetime and the Doppler broadening of the emitted radiation averages out. As a result a sharp line with the natural linewidth $\Gamma=\hbar / \tau$ at the position $E_{0}$ is emitted from the source and nuclear resonance absorption with an energetic resolution $\Gamma / E_{0}=10^{-13}$, suf- ficient to resolve hyperfine interactions, is possible [2]. The above mentioned conditions for recoilless emission limits the Mössbauer isotopes to those isotopes which possess low energy nuclear $\gamma$-transitions (small recoil moment), long lifetimes of the excited state (hyperfine resolution), high Debye temperature (small $\Delta x$ ) and solid sources and absorbers. These conditions are ideally fulfilled for ${ }^{57} \mathrm{Fe}$ which is indeed the most popular Mössbauer isotope. Fig. 1 shows schematically the decay scheme of ${ }^{57} \mathrm{Co}$, which is used as parent for a ${ }^{57} \mathrm{Fe}$ Mössbauer source. The Mössbauer resonant absorption and varios re-emissions are also indicated in Fig.1. In order to vary the energy of the Mössbauer radiation emitted by the source in the range of hyperfine energies and thus render possible Mössbauer spectroscopy it is sufficient to move the source with some $\mathrm{mm} / \mathrm{s}$. The intensity of the Mössbauer photons behind the absorber as function of the relative velocity between source and absorber represents a Mössbauer (transmission) spectrum as shown schematically in the insert of Fig.1.

\section{I.2 Hyperfine Interactions}

The interaction of the extended nuclear charge with electrons present in the nuclear volume leads to a shift of the center of the spectrum (isomer shift) and provides information on the valence state of the Mössbauer atom. 

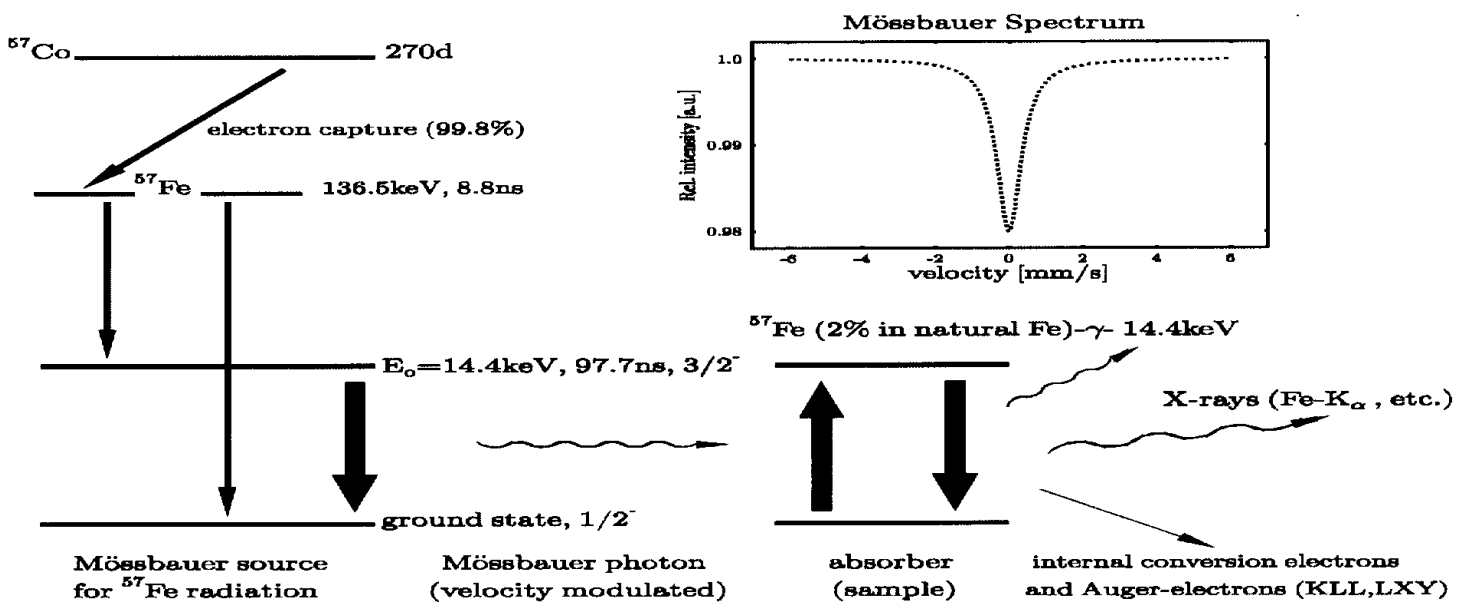

Figure 1. Decay scheme of ${ }^{57} \mathrm{Co}$ and Mössbauer resonance absorption and re-emission of ${ }^{57} \mathrm{Fe}$. Insert: Mössbauer transmission spectrum.

An electric field gradient present at the nucleus' site interacts with the nuclear electric quadrupole moment and results in a (quadrupole) splitting of a single Mössbauer line (into two lines for nuclei with nuclear spin of the ground and excited states, $I_{g, e}=1 / 2,3 / 2$, respectively). This nuclear quadrupole interaction allows conclusions on the valence, bonding properties and symmetry of the vicinity of the Mössbauer atom.

The magnetic hyperfine interaction is especially important in our context. The main part of it stems from the Fermi contact interaction which is the interaction of the nuclear magnetic dipole moment with a net spinup or down s-electron density at the nucleus. It can be represented by a spin-Hamiltonian [2]

$$
H_{m h f}=\vec{S} \stackrel{\leftrightarrow}{A} \vec{I},
$$

where $\stackrel{\leftrightarrow}{A}$ is the hyperfine tensor. When the hyperfine tensor is isotropic, i.e. $H_{m h f}=A S_{z} I_{z}$ with the hyperfine interaction constant $A$, we can interpret it as a nuclear Zeeman effect due to the field $B_{n}$ at the nucleus' site (inner field)

$$
H_{m h f}=-g n \mu_{B} B_{n} I .
$$

For a Mössbauer isotope with $I_{g, e}=1 / 2,3 / 2$ a sixline spectrum with relative intensities depending on the angle between the $\gamma$-direction and the $z$-axis results. The presence of six lines in a ${ }^{57} \mathrm{Fe}$ Mössbauer spectrum thus indicates the presence of a (static) magnetic field at the nucleus which may be caused by long range order magnetism.

\section{I.3 Fluctuating hyperfine fields}

Fluctuations of the hyperfine interactions can alter the spectra. E.g. when $B_{n}$ changes it's direction many times during the lifetime of the excited state the nucleus "sees" only an average zero field and the splitting collapses.
It can be shown that the Mössbauer absorption spectrum $I(\omega)$ observed in the energy (frequency) domain, i. e. the number of counts in transmission direction behind the absorber as function of the source velocity $v$, is given by the real part of the trace of the spectrum of the temporal correlation function of the Mössbauer radiation operator $A$ (e.g. magnetic dipole radiation) as $[3,4]$

$$
I(\omega)=\frac{2}{\Gamma} \operatorname{Re} \int_{0}^{\infty} d t e^{-p t} \operatorname{Tr}\left(\rho A(t) A^{+}(0)\right),
$$

where $p=\Gamma / 2-i \omega\left(E_{0}=\hbar \omega=E_{0} v / c\right)$ and $\rho$ is the density operator of the whole system. It is advantageous here to use superoperators and Hamilton superoperators $\mathbf{H}^{\mathbf{x}}$ (Liouville operators) in order to avoid time ordering problems when $H(t)$ is not commuting with itself at different times as is the case when combined nuclear quadrupole and (non-diagonal) magnetic hyperfine interaction is present. A superoperator $\mathbf{A}^{\mathbf{x}}$ acts on another operator $B$ in the form $\mathbf{A}^{\mathbf{x}} B=A^{+} B-B A$ which is equal to the commutator $[A, B]$ when the operator $A$ is hermitian. The Liouville operators can be represented in a projector base $\mid i j)=|i><j|$ of the Hamiltonian eigenstates $|i\rangle$ and $|j\rangle$ and the eigenvalues of $\mathbf{H}^{\mathbf{x}}$ are the spectroscopic important differences $\left(E_{i}-E_{j}\right)$. The product $(B \mid A)$ is given by $(B \mid A)=$ $\operatorname{Tr}(B+A)$ and the time evolution $A(t)$ of an operator $A$ can be written $\exp (i H t) A \exp (-i A t)=\exp \left(i \mathbf{H}^{\mathbf{x}} t\right) A(0)$. We can then write [4]

$$
\begin{gathered}
I(\omega)=\frac{2}{\Gamma} \operatorname{Re} \operatorname{Tr}\left(\rho A \frac{1}{p-i \mathbf{H}^{x}} A^{+}\right) \\
=\frac{2}{\Gamma} \operatorname{Re} \operatorname{Tr} \int_{0}^{\infty} d t e^{-p t} \rho e^{i \mathbf{H}^{x} t} A(0) A^{+}(0),
\end{gathered}
$$

Here a practical difficulty arises: $\mathbf{H}^{\mathbf{x}}$ comprises the entire solid (nucleus-electrons-phonons- photons and interactions) and the calculation of $I(\omega)$ involves the in- 
version of matrices with huge dimensions. Two simplifying approaches have been used: the stochastic and ab initio models. We present here some ideas on the latter model.

The Hamiltonian of the system is subdivided

$$
H=H_{\text {Ion }}+H_{\text {Bath }}+H_{\text {Ion-Bath }} .
$$

One shows then that under the assumptions $\mathbf{H}^{\mathbf{x}}$ Bath-Ion $<<H_{\text {Ion }}, H_{\text {Bath }}$ (convergency of approximation solution) and $\rho=\rho_{\text {Ion }} \cdot \rho_{\text {Bath }}$ (irreversible Bath-Ion interaction) the Mössbauer spectrum is given by $[4,5]$

$$
I(\omega) \propto \operatorname{ReTr}_{\text {Ion }}\left(\rho_{\text {Ion }} A\left[p-i \mathbf{H}_{\text {Ion }}^{\mathbf{x}}-\mathbf{R}(p)\right]^{-1} A^{+}\right),
$$

where the relaxation superoperator $\mathbf{R}$ contains matrix elements of the spin-operator $S_{q}$ and bath correlation spectral densities which depend on bath-operators $F_{q}$. Here the ion-bath interaction was described by a $d y$ namical spin Hamiltonian $H_{\text {Ion-Bath }}=\sum_{q} S_{q} F_{q} . E . g$. in $2^{\text {nd }}$ order of $\mathbf{H}^{\mathbf{x}}$ Ion-Bath after some tedious calculation one obtains as relaxation supermatrix elements $[5]$

$$
\begin{aligned}
\left.(g e)|R| g^{\prime} e^{\prime}\right) & =\sum_{q, q^{\prime}}\left[\delta_{g g^{\prime}} \sum_{e^{\prime \prime}}<e^{\prime}\left|S_{q^{\prime}}\right| e^{\prime \prime}><e^{\prime \prime}\left|S_{q}\right| e>I_{q^{\prime} q}^{\prime}\left(\omega_{g}-\omega_{e}+i p\right)\right. \\
& +\delta_{e e^{\prime}} \sum_{g^{\prime \prime}}<g^{\prime}\left|S_{q}\right| g^{\prime \prime}><g^{\prime \prime}\left|S_{q^{\prime}}\right| g>I^{\prime \prime}{ }_{q^{\prime} q}\left(\omega_{g} "-\omega_{e}+i p\right) \\
& -<e^{\prime}\left|S_{q}\right| e><g\left|S_{q}\right| g^{\prime}>I^{\prime \prime}{ }_{q^{\prime} q}\left(\omega_{g^{\prime}}-\omega_{e}+i p\right) \\
& \left.-<g\left|S_{q^{\prime}}\right| g^{\prime}><e^{\prime}\left|S_{q}\right| e>I^{\prime \prime}{ }_{q^{\prime} q}\left(\omega_{g}-\omega_{e^{\prime}}+i p\right)\right]
\end{aligned}
$$

with the reservoir correlation spectral densities

$$
\begin{gathered}
I_{q^{\prime} q}^{\prime}(\Omega)=\int_{0}^{\infty} e^{i \Omega t} \operatorname{Tr}_{\mathrm{Bath}}\left[\rho_{\mathrm{Bath}} F_{q^{\prime}}^{I}(t) F_{q}^{I}(0)\right] d t \\
I_{q^{\prime} q}^{\prime \prime}(\Omega)=\int_{0}^{\infty} e^{i \Omega t} \operatorname{Tr}_{\mathrm{Bath}}\left[\rho_{\mathrm{Bath}} F_{q}^{I}(0) F_{q^{\prime}}^{I}(t)\right] d t
\end{gathered}
$$

where the bath operators $F_{q, q]}^{I}$ are in the interaction representation. When $I_{q^{\prime} q}^{\prime}$ and $I^{\prime \prime}{ }_{q^{\prime} q}$ are independent of $\omega$ ("white noise approximation", normally valid in the case of spin-phonon relaxation), the matrix elements $(e g|R| e g)$ are transition probabilities $W_{g}^{e}$ between the states $\mid e>$ and $\mid g>$.

\section{I.4 Spin-phonon interaction}

Simplifying, we start with a dynamical spinHamiltonian which represents the spin-phonon interaction in $2^{\text {nd }}$ order perturbation theory $\left(B_{2}^{q}\right.$ are coupling constants) $[6,7]$

$$
\mathbf{H}_{\text {Bath-Ion }}=\sum_{q=-2}^{2} O_{2}^{q} B_{2}^{q}\left(\epsilon_{a v}+\epsilon_{a v}^{2}+\ldots\right)
$$

with the averaged strain operator

$$
\epsilon_{a v}=\sum_{j}\left(\frac{\hbar}{2 M \omega_{j}}\right)^{1 / 2} k_{j}\left(a_{j}^{+} e^{-i \vec{k}_{j} \vec{r}}+a_{j} e^{i \vec{k}_{j} \vec{r}}\right) .
$$

The equivalent operators $O_{2}^{q}$ contain the ladder operators $S_{ \pm}^{2}$. We take only direct processes into account (see Fig.2) where the absorption (annihilation) or emission (creation) of a phonon leads to a spin transition.

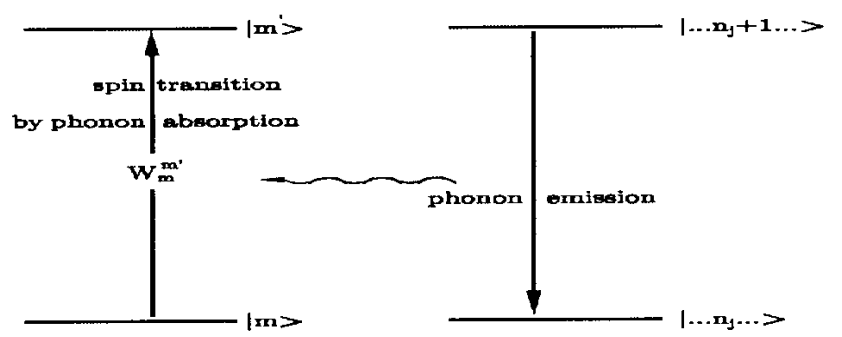

Figure 2. Spin excitation by absorption of a phonon in a direct process.

The spectral bath correlation density can then be calculated in the harmonic approximation by introducing the Debye model. In the long-wavelength limit $(\omega=v k)$ we obtain $(\rho=M / V=$ density, $v=$ averaged sound velocity) 


$$
I_{q^{\prime} q}^{\prime(2)}=I_{q^{\prime} q}^{\prime \prime(2)}=\frac{3\left(\omega_{m^{\prime}}-\omega_{m}\right)^{3} B_{2}^{q^{\prime}} B_{2}^{q}}{4 \pi \hbar \rho v^{5}} \cdot \frac{1}{e^{\hbar\left(\omega_{m^{\prime}}-\omega_{m}\right) / k_{B} T}-1}
$$

and the spin-phonon transition probabilities (cf. Fig.2) are

$$
W_{m}^{m^{\prime}}=\frac{3\left(B_{1}^{p}\right)^{2}\left|<m^{\prime}\right| O_{1}^{p}|m>|^{2}}{2 \pi \hbar \rho v^{5}} \frac{\left(E_{m^{\prime}}-E_{m}\right)^{3}}{\exp \left(\left(E_{m^{\prime}}-E_{m}\right) / k_{B} T\right)-1} .
$$

With these relations paramagnetic spin-phonon relaxation spectra of e.g. Fe(III) impurities $(S=5 / 2)$ can be calculated, eventually using effective spins - [5] because the supermatrix for $S=5 / 2$ has already a rank of $\left(2 I_{g}+1\right)\left(2 I_{e}+1\right)(2 S+1)^{2}=288$ for ${ }^{57} \mathrm{Fe}$ and is not hermitian. An example for $\mathrm{Fe}(\mathrm{III})$ impurities in monocrystals of $\mathrm{LiNbO}_{3}$ is given in [8]. For bigger spins like that corresponding to the total magnetic moment of magnetic nanoparticles $(S=1000-10000)$ the rank of the supermatrix involved would be of the order of $10^{8}$, without any possibility of numerical treatment. But it is yet possible to realize calculations for big spins when only diagonal interaction is present and thus no superoperator formalism is needed for the combined stochastic-quantum mechanical problem (see be below in section II.4).

\section{Superparamagnetism}

Superparamagnetism has to do with the thermally induced inversion of the magnetization of small (nanosized) magnetic particles. This is an important phenomenon since ultra-fine magnetic particles are important in many systems, e.g. in soils, rocks, argiles, ceramics, paintings, living organisms (ferritin, magnetic bacteria, magnetically based navigation of animals), recording media (what is the ultimate magnetic storage density?), catalysts, etc. and ferrofluids.

\section{II.1 Basic ideas of superparamagnetism}

When the dimensions of a small magnetic particle are of the order of the width of a Bloch wall the particle will be a single domain particle. At low tempera- tures the spin waves are frozen and thus the magnetization is equal to the spontaneous magnetization $M_{s}$. Since the exchange (or superexchange) interaction is predominant compared to anisotropy interactions $|M|$ may be regarded as constant so that the only degree of freedom of the magnetization would be its orientation $\theta$ relative to some (uniaxial) anisotropy axis. When measuring a property related to the magnetization at $T>0$ it may happen that the magnetization fluctuates (thermally activated) between easy directions $\underline{a n d}$ the characteristic time of measurement is greater than the (overbarrier) fluctuation time of the magnetization. In this case we say that superparamagnetic behavior is measured (the measurement method "sees" only the time-averaged value). From now on we will use the following simplifications: only an uniaxial symmetry of the form [9-12]

$$
E(\theta)=K V \sin ^{2} \theta-M_{s} V B \cos \theta
$$

is present ( $K=$ anisotropy constant, $V=$ volume of the particle), the external field $B$ is parallel to the positive $z$-direction, all particles are identical in material, form and spatial orientation and are uniform (no surface effects) and isolated (no particle-particle interaction) and the rotation of the magnetization happens by coherent rotation of the spins ("unison"). Under these circumstances various authors deduced expressions for the relaxation time $\tau$ of reversions of the magnetization between the easy $\pm z$-directions.

Néel [11] relates the vibrational strain energy of the particles to its thermal energy and calculates the magnetic energy caused by the deformation. At low temperature he obtains

$$
\tau_{ \pm \text {Néel }}^{-1}=(2 K / \pi G)^{1 / 2} \gamma_{0} M_{s}^{-1}\left|3 G \lambda+D M_{s}^{2}\right| \alpha^{1 / 2}\left(1-h^{2}\right)^{1 / 2}(1 \pm h) \exp \left(-\alpha(1 \pm h)^{2}\right),
$$


where $G$ is Young's modulus, $\gamma_{0}=g \mu_{B} / \hbar \approx g e / m$ is the gyromagnetic ratio, $\alpha=K V / k_{B} T$ and $h=$ $B M_{s} / 2 K$ is the reduced external field $(0 \leq h \leq 1)$.

Brown [12] considers a random walk of the magnetization on a sphere similar to Brownian motion. In the classical equation of motion of the magnetization (Gilbert's equation) a dissipative and random field term $h(t)$ is introduced. A Fokker-Planck equation is obtained which is approximately solved. The result for low temperature $(\alpha<<1)$ is

$\tau_{ \pm \mathrm{Br}}^{-1} \approx\left(\gamma_{0} K / \pi^{1 / 2} M_{S}\right) \alpha\left(1-h^{2}\right)(1 \pm h) \exp \left(-\alpha(1 \pm h)^{2}\right)$

This expression is widely used in the literature but it's not valid for high temperatures and $h \approx 1$.

Bessais, Ben-Jaffel, Dormann [13] find more exact solutions of the Fokker-Planck equation by using Fourier and Chebyshev series and deduce

$$
\tau_{\mathrm{BBD}}^{-1}=K \gamma_{0} M_{S}(1+\alpha / 4)^{5 / 2} \alpha^{-1} \exp (-\alpha) .
$$

Villain et al. [14] analyze paramagnetic like relaxation in magnetic molecules with $\mathrm{S}=10$ due to spinphonon interaction. The corresponding relaxation rate was calculated to

$$
\tau_{\mathrm{V}}^{-1} \approx 3 B^{2}(K V)^{3}\left(2 \pi \hbar^{4} \rho v^{5} \alpha S^{4}\right)^{-1} \exp (-\alpha) .
$$

Jones, Srivastava [15] introduce the "many states model" in order to calculate Mössbauer spectra (see below in section II.4). For a large (classical) spin (continuum limit) they also deduce from this model a FokkerPlanck like equation and solve it in the low temperature regime and obtain $(R \propto$ square of a random field)

$$
\tau_{ \pm \mathrm{JS}^{-1}} \approx R \pi^{1 / 2} \alpha^{3 / 2}\left(1-h^{2}\right)(1 \pm h) \exp \left(-\alpha(1 \pm h)^{2}\right)
$$

One observes that all these expressions contain the same Arrhenius-like exponential factor but various prefactors. In principle they are only valid in the low temperature regime. No microscopic, nonphenomenological explanation of the superparamagnetic mechanism is given by the above authors.

\section{II.2 Superparamagnetism by spin- phonon relaxation}

Recently a mechanism for superparamagnetic relaxation based on spin-phonon interaction was suggested [16]. We shortly repeat here the main ideas and results of this work.

The supposed unison rotation of the spins suggests the attribution of a large spin to the magnetization defined by $M_{s} V=g \mu_{B} S$. Relating $S \cos \theta$ to the spin operator $S_{z}$ and suppressing a constant term $K V$, the anisotropy energy (13) corresponds to a spinHamiltonian

$$
H_{s p}=-A S_{z}^{2}-g \mu_{B} B S_{z},
$$

where $g$ is the electronic $g$-factor, $\mu_{B}$ the Bohr magneton and $A S^{2}=K V$ (height of the barrier for $B=0$ ). The superparamagnetic relaxation of the particles is assumed to be induced by interaction of the large spin $S$ with the phonons of the particle (or of a bath). In order to turn over between opposite easy directions the spin $S$ must pass through intermediate levels characterized by $S_{z}$. Eigenvectors of (19) are $\left|S_{z}\right\rangle=|m\rangle$, with eigenvalues $E_{m}=-A S_{z}^{2}-2 A h S S_{z}$ for $S_{z}=$ $-S,-S+1, \ldots, S-1, S$. Fig. 3 shows a graph of $E_{m}$ vs. $m$ and a possible phonon induced spin transition between the lowest levels of both sides of the barrier.

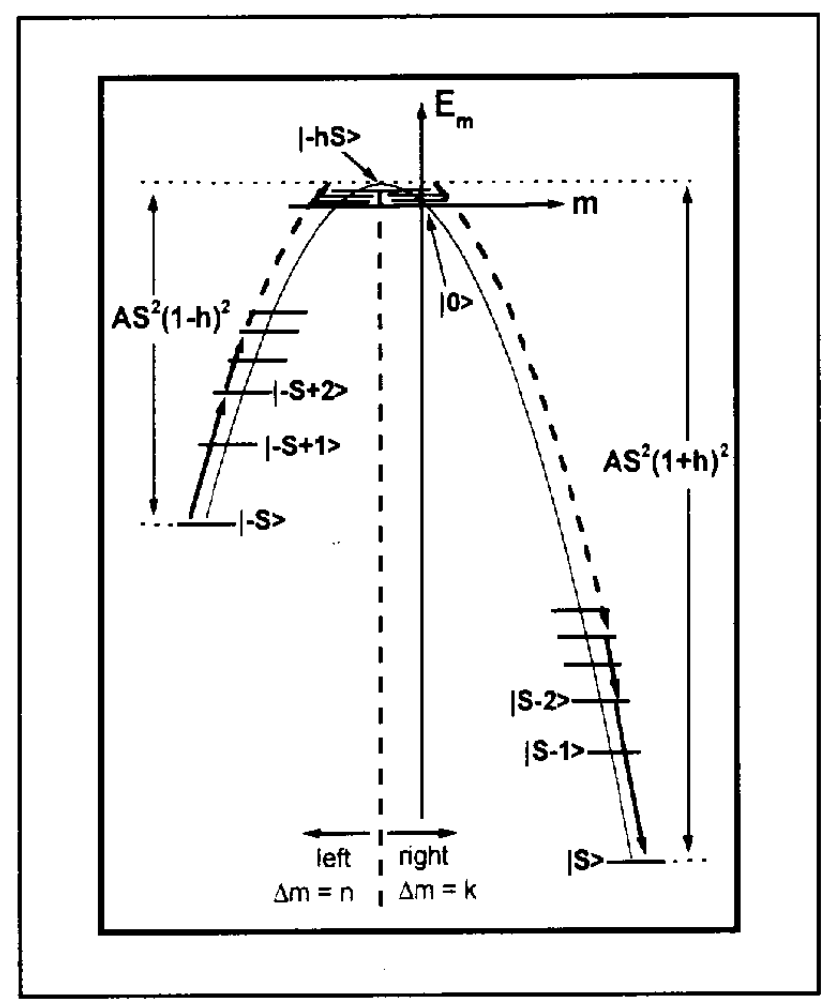

Figure 3. Anisotropy energy vs. eigenvalues with applied magnetic field. Spin transitions from $\mid-S>$ to $\mid S>$ with $\Delta m=\Delta n=2$ are indicated.

\section{II.3 Calculation of a relaxation time for} $|S>\leftrightarrow|-S>$

The spin transition rate induced by phonons is given in (12). Considering only the relaxation $|S\rangle \leftrightarrow|-S\rangle$ under the condition that the occupation of all intermediate levels remains constant (pseudo two level system) and assuming detailed balance, a rate equations system (master equations) can be established and solved for a relaxation time $\tau_{ \pm}$[16]. In the case of spin jumps with $\Delta m=\Delta n=2$ we obtain $\left(C^{\prime}=\right.$ $\left.\left(3\left(B_{1} p\right)^{2} / 2 \pi \hbar^{4} \rho v^{5}\right)\left(A S^{2}\right)^{3}, \alpha_{ \pm}=\alpha(1 \pm h)^{2}\right)$

$$
\tau_{ \pm}^{-1}=C^{\prime-1} \exp \left(\alpha_{ \pm}\right)(S / 2)^{6}(\sigma(+h)+\sigma(-h))
$$

with 


$$
\sigma( \pm h)=\sum_{i=0}^{\frac{S}{2}(1 \pm h)-1} \frac{\exp \left(-\alpha\left(\frac{2}{S}\right)^{2} i^{2}\right)\left(1-\exp \left(-\alpha\left(\frac{2}{S}\right)^{2}(1+2 i)\right)\right)}{\left.(1+2 i)^{3}(S(1 \pm h-2 i)(S(1 \pm h)-2 i-1) D) 1 \mp h\right)+2 i+2(S(\mp h)+2 i+1)} .
$$

A good approximation up to $h \approx 0.95$ is

$$
\tau_{ \pm}^{-1} \approx 5 C^{\prime} \alpha^{-1}\left(1-h^{2}\right)^{2} \exp \left(-\alpha_{ \pm}\right) \approx 2 \cdot 10^{13} s^{-1} \alpha^{-1}\left(1-h^{2}\right)^{2} \exp \left(-\alpha_{ \pm}\right) .
$$

Here $S=3222, \rho=5000 \mathrm{kgm}^{-3}, v=3000 \mathrm{~ms}^{-1}$ and $K V=A S^{2}=2.67 \cdot 10^{-20} \mathrm{~J}$ was assumed, which are typical values for a $90 \AA \mathrm{MnFe}_{2} \mathrm{O}_{4}$-particle with 5 spins per unit cell of $8.4 \AA$ lattice constant $[16,17]$. For the only free parameter, the coupling parameter $B_{2}^{2}$, a value of $13 \mathrm{~cm}^{-1}$ was chosen, which results in relaxation rates in accordance with the experiments.

The functional dependence on the anisotropy energy $K V$, temperature $T$ and external field $(h)$ in the expressions (14)-(18) and (21) is similar. They contain the same Arrhenius-like factor and differ only slightly in the field dependence of the pre-factor, which is proportional to $\left(1-h^{2}\right)(1 \pm h)$ in (15) and (18) instead of $\left(1-h^{2}\right)(1+h)(1-h)$ in $(21)$. In view of the completely different physical approach used this may be remarkable. Of course all the expressions differ in the other factors which appear in the pre-factor since they depend on the parameters involved in the specific model. We conclude that our model adequately describes superparamagnetism.

On the basis of (21) Mössbauer spectra can be calculated using an effective spin. At low temperature experimental spectra can be reproduced [17]. Spectra at somewhat higher temperature can approximately reproduced taking collective excitations, i. e. a thermally averaged inner field, into account. However for higher temperatures this method fails and calculations based on the method presented in the following are indicated.

\section{II.4 Multi-level calculation of Mössbauer spectra}

In the case of isotropic magnetic hyperfine interaction and diagonal nuclear Hamiltonian (nuclear quadrupole interaction $<<$ magnetic hyperfine interaction) the expression (6) and (7) can be simplified so that no superoperators are needed. Furthermore one can simulate a Mössbauer spectrum by summing up three separately calculated spectra consisting of the pairs of lines (1-6), (2-5), (3-4) in the six-lines spectrum. The simplified expression [15] for one pair of lines reads

$I(\omega) \propto 2 \operatorname{Re}\left(\vec{W} \overleftrightarrow{M}^{-1} \vec{I}\right)$ whit $\overleftrightarrow{M}=\left(i\left(\omega-\omega_{i}\right)+\Gamma\right) \overleftrightarrow{1}-\overleftrightarrow{R}$

where $\stackrel{\leftrightarrow}{W}$ is a row vector consisting of probabilities (Boltzmann factors) of the $(2 S+1)$ states and $M$ contains the static line positions $\omega_{i}$, given by the splitting (1-6), which is assumed to be proportional to $S_{z}$. The relaxation matrix $\stackrel{\leftrightarrow}{R}$ has as elements transition probabilities $r_{i j}=W_{i j}$ between the states $|i>\rightarrow| j>(i \neq j)$ and $r_{i i}=-\sum_{j} W_{I}^{j}(i \neq j)$. The total Mössbauer spectrum is obtained by summing up the three calculated two-lines spectra (1-6), (2-5) and (3-4), where in the calculation of the $\omega_{i}$ adequately reduced splittings (2-5) and (3-4), still proportional to $S_{z}$, and the same transition probabilities as in the calculation of (1-6) are used.

As before, for the spin-transitions $\Delta S_{z}=2$ is presumed. We take therefore only spin-matrix elements, that contain $S_{ \pm}^{2}$, into account, which results in transition probabilities

$$
\begin{aligned}
& W_{S_{z}}^{S_{z} \mp 2}=C\left(B / \mathrm{cm}^{-1}\right)^{2} S^{4}\left(1 \pm \frac{S_{z}}{S}\right)\left(1 \pm \frac{S_{z}}{S}-\frac{1}{S}\right)\left(1 \mp \frac{S_{z}}{S}+\frac{1}{S}\right)\left(1 \mp \frac{S_{z}}{S}+\frac{2}{S}\right) \frac{(4 A S)^{3}\left(-\frac{1}{S} \pm \frac{S_{z}}{S} \pm h\right)^{3}}{e^{\frac{4 A S}{k^{T} T}\left(-\frac{1}{S} \pm \frac{S_{z}}{S} \pm h\right)}-1} . \\
& S_{z}= \pm S, \ldots, \mp S \pm 2
\end{aligned}
$$


The Boltzmann factors contained in $\stackrel{\leftrightarrow}{W}$ are

$$
W_{x} \propto \exp \left(-\frac{K V}{k_{B} T}\left((1+h)^{2}-\left(\frac{S_{z}}{S}+h\right)^{2}\right)\right)
$$

Introducing these expressions in (22) one can simulate Mössbauer spectra. The inverse of $\overleftrightarrow{M}$ is required for each value of $\omega$ (256 Mössbauer velocities), involving a order of 256 times $N^{3}$ operations for a matrix of rank $N$. In our case $\stackrel{\leftrightarrow}{M}$ is tridiagonal and thus only of the order of $256 \mathrm{~N}^{2}$ operations are necessary for inversion. A further significant saving in computer time is obtained by observing that in (22) in fact not the inverse $\overleftrightarrow{M}^{-1}$ but only $\overleftrightarrow{M}^{-1} \overrightarrow{1}$ is needed. Writing $\overleftrightarrow{M}^{-1} \overrightarrow{1}=\vec{V}$ or the $\overleftrightarrow{M} \vec{V}=1$ problem is reduced to solve a set of simultaneous linear equations for which requires only an order of $256 \mathrm{~N}$ operations $[15,18]$. This method allows one to simulate spectra for spins $\mathrm{S}$ up to several thousand, corresponding to spins found in real systems, on a modest computer in a few minutes.

Fig. 4 shows some simulations of spectra using this multi-level relaxation method for a spin $S=3222$ at different temperatures and several external magnetic fields. The parameters used in the calculations of the transition probabilities are that of eq. (21).

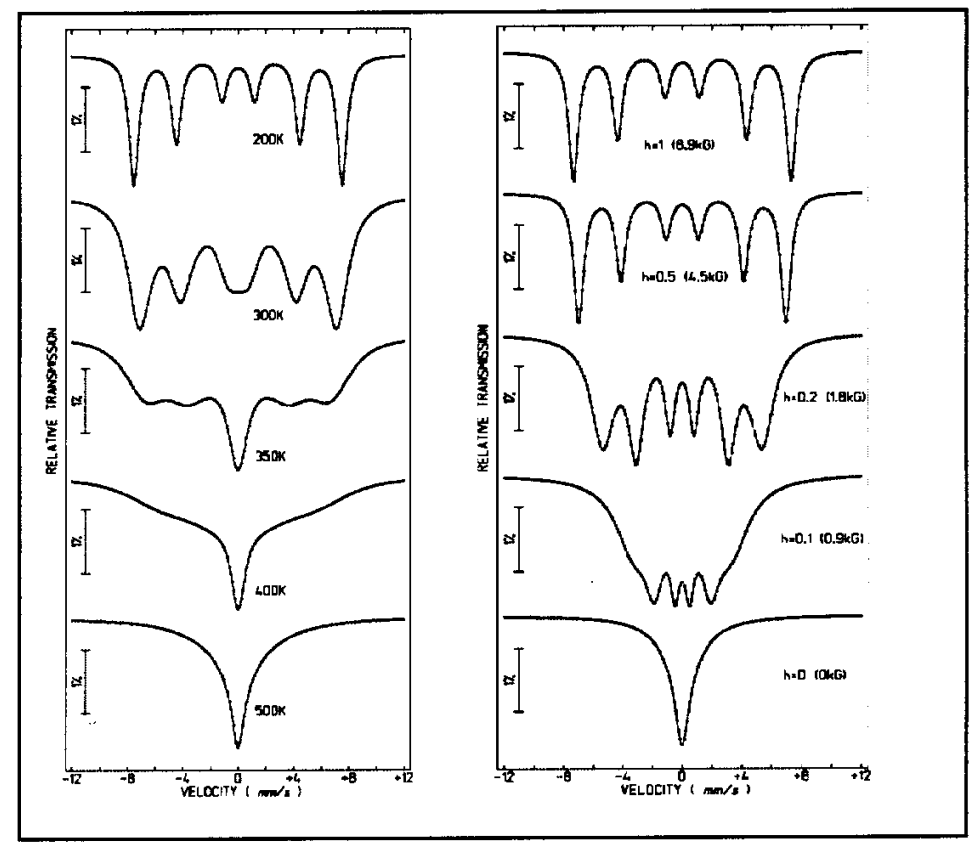

Figure 4. Simulated Mössbauer spectra at different temperatures and zero external field (left) and different fields at $600 \mathrm{~K}$ (right). See text.

Despite the above described possibility of rapid simulation of spectra for a large spin it may sometimes be necessary to use a smaller (reduced) spin in order to calculate real spectra. E. g., if the simulation of powder spectra is desired, one has to effect an average over the different orientations of the crystallites relative to the external field- and $\gamma$-direction. This implies in the presence of a field component perpendicular to the $z$ axis, which results in a mixture of the spin levels, so that $\overleftrightarrow{M}$ is no longer tridiagonal. Furthermore, when size-dispersion is present, as is always the case in real samples, one has also to sum up sufficiently spectra corresponding to different volumes (and anisotropy constant values $K$, which often depend on the crystallite size).
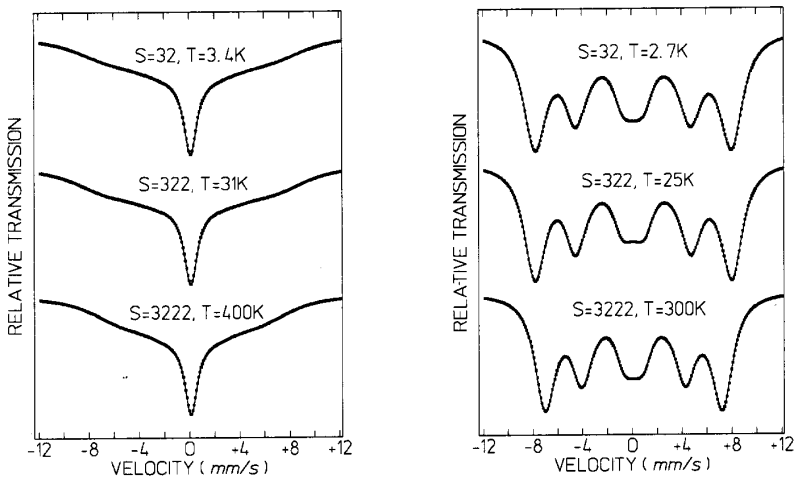

Figure 5. Variation of $S, S=3222,322,32$ (simulation time $=5 \mathrm{~min}, 20 \mathrm{~s}, 3 \mathrm{~s})$. 
We compared therefore spectra simulations for different values $S_{\text {red }}$ of reduced spins at different temperatures. The result is shown in Fig. 5. It can be seen that practically identical spectra are obtained for different spins when the temperature is reduced by a factor of approximately $S / S_{\text {red }}$. One can therefore use a smaller spin $S_{\text {red }}$ in practical simulations.

\section{Application to ferrofluids}

Many ferrofluids are based on nano-sized particles of ferrites. Static Mössbauer spectra of ferrites can normally be satisfactorily fitted by two sextets with relative total area corresponding to the ratio of ${ }^{57} \mathrm{Fe}$ atoms on octahedral and tetrahedral substitutional sites (Néel model [19]). However, for simplification of the calculations, we use in the following simulations only one single sextet with larger $\left(\sim 0.4 \mathrm{mms}^{-1}\right)$ linewidth and suppress also the quadrupole splitting. In the calculations also the size distribution (log-normal distribution with mean diameter $d_{0}$ and width $\sigma$ ) as deduced from the sample synthesis and electron microscopy has been taken into account by summing up spectra (sample synthesis by F.A. Tourinho, UnB, Brasilia). The random orientation of the nanoparticles has been counted for by using relative line intensities in the sextets of $3: 2: 1$. Fig. 6 shows experimental spectra of a $\mathrm{NiFe}_{2} \mathrm{O}_{4}$ based frozen ferrofluid in the temperature range $T=90 \ldots 4.2 \mathrm{~K}$. In the corresponding simulations a reduced spin $S_{\text {red }}$ depending on the crystallite diameter d (size distribution) defined by $S_{\text {red }}(d)=S\left(d_{0}\right)\left(d / d_{0}\right)^{3}$ with $S\left(d_{0}\right)=32$ and reduced temperatures $T_{\text {red }}=T\left(S_{\text {red }} / 3222\right)$ were used.

Similarly, Fig. 7 shows experimental spectra and simulations of a powder of $\mathrm{CoFe}_{2} \mathrm{O}_{4}$, precursor of a cobaltferrite based ferrofluid. The simulations have been done analogously to those of Fig.6. Considering the various simplifications made the coincidence of experimental and simulated spectra is satisfactory.

\section{Conclusion}

Experimental superparamagnetic Mössbauer line patterns are reproduced by ab-initio simulations based on a spin-phonon interaction model and taking intermediate spin levels into account, without using a thermallly averaged hyperfine field due to collective excitations. We use spin-transitions probabilities per time $W_{i}^{j}$ derived from a dynamic spin-Hamiltonian (weak spinbath interaction) that induces transitions in the spin and phonon subsystems and adopt the Debye model in the long wavelength limit.

With this method one is able to calculate Mössbauer spectra for $S=3222$ within several minutes (provided that the relaxation matrix $\mathbf{R}$ is tridiagonal) on a small personal computer. Comparison of spectra calculated with a smaller spin exhibit similar shapes than that of the "real" spin $S$, provided the temperature is adequately reduced. Simulations of Mössbauer spectra of ferrofluids-ferrites are compared with real spectra and show good correspondence.

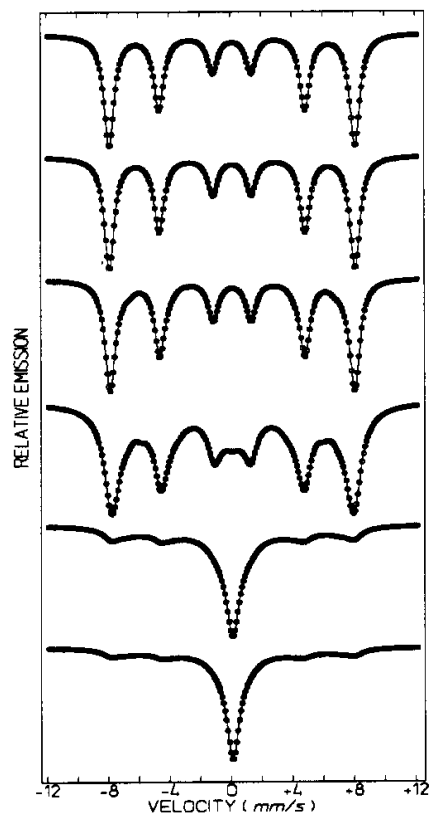

Figure 6. Spectra of frozen $\mathrm{NiFe}_{2} \mathrm{O}_{4}$-ferrofluid, $d_{0}=10 \mathrm{~nm}$ (left) and simulations with $S_{\text {red }}\left(d_{0}\right)=32$ and reduced temperatures, log-normal size distribution $\left(\sigma=0.1, d_{0} 6=10 \mathrm{~nm}\right), K=4 \cdot 10^{4} \mathrm{Jm}^{-3}$, no quadrupole interaction. 

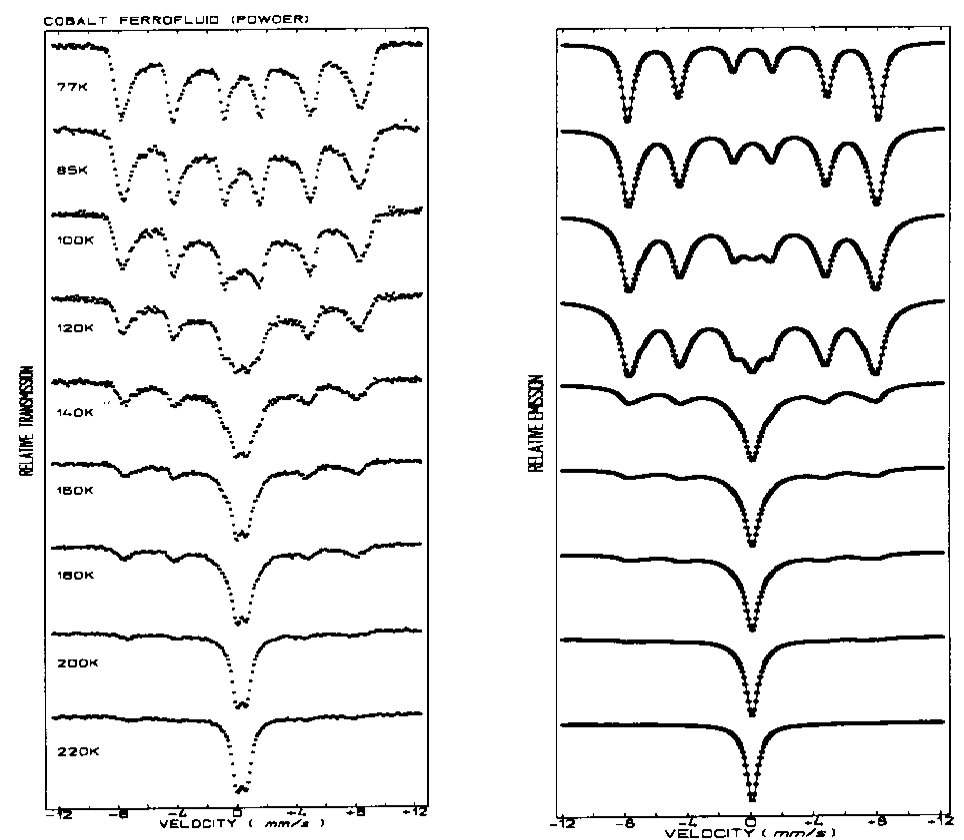

Figure 7. $\mathrm{CoFe}_{2} \mathrm{O}_{4}$-powder spectra, $d_{0}=4.3 \mathrm{~nm}$ (left). $\mathrm{CoFe}_{2} \mathrm{O}_{4}$-simulations, $d_{0}=5 \mathrm{~nm}, \sigma=0.1, S\left(d_{0}\right)=32, K=1.6 \cdot 10^{5} \mathrm{Jm}^{-3}$, (right)

\section{References}

[1] R.L. Mössbauer, Z. Physik 151, 124 (1958); Naturwissenschaften 45, 538 (1958); Z. Naturforsch. 14a, 211 (1959).

[2] V.I. Goldanskii, R.H. Heber, Chemical applications of Mössbauer Spectroscopy, Academic Press, New York, 1968.

[3] S. Dattagupta, G.K. Shenoy, B.D. Dunlap and L. Asch, Phys. Rev. B16, 3893 (1977).

[4] L. Cianchi, P. Moretti, M. Mancini and G. Spina, Rep. Progr. Phys. 49, 1243 (1986).

[5] H.D. Pfannes, Hyp. Interact. 110, 127 (1997).

[6] R. Orbach, H.J. Stapleton, in: Electron Paramagnetic Resonance, ed. S. Geschwind (Plenum Press, New York, 1972).

[7] K.K.P. Srivastava, S.N. Mishra, Phys. Stat. Sol. (b) 100, 65 (1980).

[8] H.-D Pfannes, R. Magalhães-Paniago, E. Bill, A. X. Trautwein, Hyp. Interact. 94, 2005 (1994).

[9] S. M $\phi$ rup, J. A. Dumesic, H. Tops $\phi$ e, in:Applications of
Mössbauer Spectroscopy, Vol. II, ed. R. L. Cohen (Academic Press, New York, 1980).

[10] J. L. Dormann, Revue Phys. Appl. 16, 275 (1981).

[11] L. Néel, Ann. Geophys. 5, 99 (1949).

[12] W.F. Brown, Jr., Phys. Rev. 130, 1677 (1963).

[13] L. Bessais, L. Ben Jaffel, J. L. Dormann, Phys. Rev. B45, 7805 (1992).

[14] J. Villain, F. Hartmann-Boutron, R. Sessoli, A. Rettori, Europhys. Lett. 27, 159 (1994).

[15] D.H. Jones, K.K.P. Srivastava, J. Mag. Magn. Mat. 78, 320 (1989).

[16] H.-D. Pfannes, A. Mijovilovich, R. Magalhães-Paniago and R. Paniago, Phys. Rev. B 62, 3372 (2000).

[17] H.D. Pfannes, J.H. Dias Filho, J.L. López, S.L. Pereira, P.C. Morais, F.A. Tourinho, Hyp. Interact. 113, 507 (1998).

[18] H.-D. Pfannes, J. H. Dias Filho, R. Magalhães-Paniago and R. Paniago, J. Mag. Magn. Mat. (2001) in the press.

[19] L. Néel, Ann. Phys. 3, 137 (1948). 